\title{
BIBLIOGRAPHY
}

1. J. J. Dennis and H. S. Wall, The limit-circle case for a positive definite J-fraction, Duke Math. J. vol. 12 (1945) pp. 255-273.

2. E. Hellinger and H. S. Wall, Contributions to the analytic theory of continued fractions and infinite matrices, Ann. of Math. (2) vol. 44 (1943) pp. 103-127.

3. J. F. Paydon and H. S. Wall, The continued fraction as a sequence of linear transformations, Duke Math. J. vol. 9 (1942) pp. 360-372.

4. W. T. Scott and H. S. Wall, A convergence theorem for continued fractions, Trans. Amer. Math. Soc. vol. 47 (1940) pp. 155-172.

5. - On the convergence and divergence of continued fractions, Amer. J. Math. vol. 69 (1947) pp. 551-561.

6. T. J. Stieltjes, Recherches sur les fractions continues, Oeuvres, vol. 2, pp. 402-566.

7. H. S. Wall and Marion Wetzel, Quadratic forms and convergence regions for continued fractions, Duke Math. J. vol. 11 (1944) pp. 89-102.

8. E. B. Van Vleck, On the convergence of continued fractions with complex elements, Trans. Amer. Math. Soc. vol. 2 (1901) pp. 205-233.

The University of Texas

\section{REMARKS ON THE NOTION OF RECURRENCE}

\section{J. WOLFOWITZ}

We give in several lines a simple proof of Poincaré's recurrence theorem.

TheOREM. Let $\Omega$ be a point set of finite Lebesgue measure, and T a one-to-one measure-preserving transformation of $\Omega$ into itself. ${ }^{1}$ Let $B \subset A \subset \Omega$ be measurable sets such that, if $b \in B, T^{n} b \notin A$ for all positive integral $n$. Then the measure $m(B)$ of $B$ is 0 .

Proof. First we show that, if $i<j,\left(T^{i} B\right)\left(T^{\prime} B\right)=0$. Suppose $c \in T^{i} B$; then from the hypothesis on $B$ it follows that $j$ is the smallest integer such that $T^{-i} c \in A$. Hence $c \notin T^{i} B$. Now if $m(B)=\delta>0, \Omega$ would contain infinitely many disjunct sets $T^{n} B$, each of measure $\delta$. This contradiction proves the theorem.

The following generalization of the above theorem is trivially obvious: The result holds if we replace the hypothesis that $T$ is measure-preserving by the following: If $m(D)>0, \lim \sup _{i} m\left\{T^{i}(D)\right\}$ $>0$.

Received by the editors April 3, 1948.

${ }^{1}$ For a discussion in probability language see $\mathrm{M}$. Kac, On the notion of recurrence in discrete stochastic processes, Bull. Amer. Math. Soc. vol. 53 (1947) pp. 1002-1010. 
Another obvious generalization is this: Let $C$ be the set of all points $c$ of $A$ such that $T^{n} c \in A$ for only finitely many $n$. Then $m(C)=0\left(\right.$ for $\left.C \subset \sum_{l=0}^{\infty} T^{-l} B\right)$.

The following is a simple derivation of Kac's theorem on the mean recurrence time. ${ }^{2}$

TheOREM. Let $T$ above be metrically transitive. Let $a \in A-B$, and $n(a)$ be the smallest positive integer such that $T^{n} a \in A$. Let $m(A)>0$. Then

$$
\int_{A-B} n(a) d m=m(\Omega) .
$$

Proof. Define $A_{k}=\{n(a)=k\}$. Let $i<j, i^{\prime}<j^{\prime}, j \neq j^{\prime}$. We notice: (a) $\left(T^{i} A_{j}\right)\left(T^{i^{\prime}} A_{j^{\prime}}\right)=0$. For $T$ has a single-valued inverse and $A_{j} A_{j^{\prime}}=0$. If $T^{i} A_{j}$ and $T^{i^{\prime}} A_{j^{\prime}}$ had a point $s$ in common, then $T^{-i} s \in A_{j}$, $T^{-i^{\prime}} s \in A_{j^{\prime}}$, in violation of the definition of $j$ and $j^{\prime}$.

$$
\int_{A-B} n(a) d m=m\left(\sum_{h=1}^{\infty} \sum_{l=0}^{h-1} T^{l} A_{h}\right)
$$

(c) Metric transitivity implies that almost every point in $\Omega$ lies in some $T^{l} A_{h}$, that is, $m\left(\sum \sum T^{l} A_{h}\right)=m(\Omega)$.

This proves the desired result.

Columbia University

2 Kac, loc. cit. Theorem 2. 